Revista lus et Praxis, Año 20, No 2, 2014, pp. 357 - 374

ISSN 0717 - 2877

Universidad de Talca - Facultad de Ciencias Jurídicas y Sociales

"Control de fusiones y nueva guía para el análisis de operaciones de concentración:

lo positivo, lo que falta y lo que sólo el legislador puede solucionar"

Cristóbal Caviedes Paúl

Trabajo recibido el 13 de enero y aprobado el 12 de marzo de 2014

\title{
Control de fusiones y nueva guía para el análisis de operaciones de concentración: lo positivo, lo que falta y lo que sólo el legislador puede solucionar*
}

MERGER CONTROL PROCEDURES AND THE NEW GUIDE FOR MERGER ANALYSIS: WHAT'S POSITIVE, WHAT'S MISSING

AND THE THINGS THAT ONLY THE LEGISLATOR CAN SOLVE

Cristóbal CaViedes Paúl**

RESUMEN

El presente artículo pretende entregar una visión crítica de los elementos procesales de la nueva "Guía para el Análisis de Operaciones de Concentración" dictada por la Fiscalía Nacional Económica en octubre de 2012, junto con indicar aquellos aspectos deficitarios de nuestro actual sistema que sólo pueden ser resueltos a través de una reforma al Decreto Ley № 211. El trabajo toma como marco de referencia los sistemas comparados de Estados Unidos, Australia, el Reino Unido y la Unión Europea, que fueron previamente analizados en la tesis de magíster del autor.

\section{ABSTRACT}

This paper aims to give a critical view of the procedural elements contained in the new Guide for Merger Analysis issued by the Fiscalía Nacional Económica in October 2012, along with indicating the deficient aspects of our current merger control procedures that can only be solved through a reform of Decree Law 211. This document uses as its theoretical framework the merger control systems of the United States, Australia, the United Kingdom and the European Union, which were previously analyzed in the author's master's degree thesis.

\section{Palabras Clave}

Guía, Concentraciones, Sistemas de control de fusiones

KEYWORDS

Guide, Mergers, Merger control procedures

En el mes de octubre de 2012, la Fiscalía Nacional Económica "la Fiscalía" o "FNE") publicó su nueva "Guía para el Análisis de Operaciones de Con-

\footnotetext{
* El presente trabajo se basa en mi tesis de Magíster, por lo que agradezco especialmente a mi profesor guía Santiago Montt Oyarzún.

** Magíster en Derecho con Mención en Derecho Público (Universidad de Chile). Correo electrónico: cristobalcaviedes@gmail.com.
} 
centración" ("la Guía"), que reemplaza a la de 2006. Una de las principales diferencias de la actual Guía con la anterior es que, en palabras de la Fiscalía: "da cuenta no sólo de los criterios de análisis actualmente relevantes, sino que describe el procedimiento especial al que la FNE sujetará la revisión de la operación en el caso que las Partes le comuniquen, antes de materializarla, su intención de concentrarse y proporcionen información para ello."2. Es decir, no solamente hay modificaciones a los criterios sustantivos para determinar si una concentración es o no contraria a la libre competencia, sino que también establece, por vez primera, reglas procesales administrativas para tratar estos casos al interior de la FNE.

Como sabemos, el control de concentraciones no es un asunto sencillo ya que los efectos anticompetitivos de una fusión son prácticamente imposibles de deshacer una vez consumada, todo lo cual obliga a los órganos respectivos a revisarlas ex ante. Por otro lado, si bien existen concentraciones altamente anticompetitivas, la gran mayoría no lo son. Más aún, las fusiones pueden ser altamente beneficiosas para los mercados al desarrollar incrementos de eficiencia transferibles a los consumidores. En consecuencia, un buen sistema de control de fusiones debe ser capaz de impedir las operaciones anticompetitivas $y$, al mismo tiempo, permitir las procompetitivas sin imponer costos administrativos excesivos. Lamentablemente, la autoridad siempre tiene asimetrías de información respecto de los particulares, razón por la cual el diseño del procedimiento de control (que es el modo a través del cual se corrige este handicap) pasa a ser crucial. En este sentido, el análisis comparado de sistemas de control preventivo de fusiones permite determinar cuáles elementos presentes en dichos mecanismos podrían ser utilizados para mejorar el desempeño del sistema chileno. En lo que respecta a Chile, nuestra actual institucionalidad es relativamente reciente. En efecto, el máximo órgano encargado de revisar las fusiones, el Tribunal de Defensa de la Libre Competencia ("TDLC"), fue creado recién a través de la Ley $\mathrm{N}^{\circ} 19.911$ de $2003^{3}$. Nuestro sistema, establecido en los arts. $18 \mathrm{~N}^{\circ} 2$ y 31 del Decreto Ley $N^{\circ} 211$ ("D.L. $N^{\circ} 211^{\prime \prime}$ ), consiste en un procedimiento voluntario que otorga una cierta "inmunidad jurídica" (art. 32) a las operaciones notificadas, controladas y aprobadas.

En Chile no existe un tipo específico para sancionar las fusiones anticompetitivas, sino que se aplica el tipo general establecido en el art. $3^{\circ}$ del D.L.

\footnotetext{
1 Fiscalía Nacional EConómica (2012).

2 Fiscalía Nacional Económica (2012).

3 Nehme y MordoJ (2012), pp. 100-109.
} 
$N^{\circ} 211^{4}$. Del mismo modo, el procedimiento no está pensado únicamente para controlar fusiones, sino que se aplica también para conocer otro tipo de hechos, actos o convenciones que no correspondan a fusiones propiamente tales. Otras características del procedimiento de control de concentraciones chileno son: a) la posibilidad, desde el año 2009, de que tanto la FNE como cualquier legítimo interesado pueda iniciar la consulta; b) la imposibilidad de ejecutar la operación mientras dure el procedimiento; y c) la preclusión de las acciones para impugnar la operación, en caso de que ella se celebre conforme a las directrices del TDLC, a menos que surjan "nuevos antecedentes" ${ }^{1}$. Hasta la fecha, nuestro procedimiento de control de fusiones se ha aplicado a pocos casos de alta relevancia pública ${ }^{6}$.

En la actualidad, nuestro sistema ha sido sometido a una serie de críticas relativas a su diseño y funcionamiento, las que se enfocan sobre todo en: a) la posibilidad de que terceros interesados puedan iniciar el procedimiento; b) los plazos excesivamente largos de los procesos de consulta ${ }^{7}$; c) la imposibilidad de negociar, al interior del procedimiento, medidas de mitigación entre la FNE y las partes ${ }^{8}$.

Basándonos sobre todo en los aspectos cuestionables de nuestro actual sistema de control de fusiones, el presente artículo busca dar una visión crítica de la regulación procesal de la nueva Guía. Por otro lado, también se pretende identificar aquellos elementos deficitarios de nuestro sistema que, a nuestro juicio, sólo es posible resolver mediante una modificación al D.L. № 211.

Para estos efectos, el trabajo se divide en tres partes: en la primera ("lo positivo") indicamos los elementos que consideramos más valorables de la Guía, en la segunda ("lo que falta"), señalamos qué aspectos de la misma son perfectibles, y en la tercera ("lo que sólo el legislador puede solucionar") mencionamos los puntos problemáticos de la consulta del art. 31 del D.L. No 211 como mecanismo de control de fusiones que únicamente pueden solucionarse por vía legislativa, proponiendo también soluciones al respecto.

\footnotetext{
${ }^{4}$ VALDÉS (2006), pp. 535-541.

5 Nehme y Mordoj (2012), pp. 100-109.

${ }^{6}$ Nehme y Mordos (2012), pp. 100-109.

7 La tramitación de los casos "D \& S/Falabella" (Resolución NN 24/2008), "Anagrama/SQM-C" (Resolución 31/2009) y "Cencosud/Condell" (Resolución № 33/2011), entre otros, tuvo una duración de al menos ocho meses en el TDLC, más unos cuatro a seis meses ante la Corte Suprema en virtud de recursos de reclamación. Por otra parte, de acuerdo a las estadísticas del propio TDLC actualizadas al 31 de diciembre de 2013, la duración promedio de las causas ante el TDLC desde su ingreso hasta la dictación de la resolución es de 236 días corridos, lo que aproximadamente equivale a ocho meses. Nehme y Mordoj (2012), p. 114; y Tribunal de Defensa de La Libre Competencia (2013 a).

${ }^{8}$ Nehme y Mordoj (2012), pp. 100-109.
} 
Antes de comenzar, una aclaración: el marco referencial de este trabajo son los principales elementos comunes a los sistemas de control de concentraciones de Estados Unidos ("EE.UU."), Australia, Reino Unido y la Unión Europea ("UE"), los que fueron analizados en nuestra tesis de magíster ${ }^{9}$ y que, por las características de un trabajo como este, no podemos tratar in extenso aquí.

\section{Lo positivo}

Los elementos más valorables de la nueva Guía son: en primer lugar, el establecimiento de un procedimiento previo a la consulta del art. 31 del D.L. $N^{\circ} 211$; y en segundo lugar, la posibilidad de negociar ampliaciones a los plazos y medidas de mitigación.

\subsection{Establecimiento de un procedimiento previo a la consulta del art. 31 del D.L. N $^{\circ} 211$}

Uno de los elementos más relevantes para el éxito de un sistema de control de concentraciones consiste en que el procedimiento se estructure en dos etapas o fases, de manera que la primera funcione como un control preliminar de la operación en general, y la segunda como un control focalizado a los aspectos problemáticos de la misma. En efecto, en los sistemas de control de fusiones de EE.UU., Australia, Reino Unido y la UE, el hito que marca el paso desde la primera hacia la segunda etapa es la emisión, por parte de la autoridad competente, de un documento denominado "declaración de objeciones" o "pliego de cargos" en el que ésta objeta, en forma clara y precisa, determinados aspectos específicos de la fusión que son lo único analizado en la segunda etapa ${ }^{10}$.

El objeto de esta estructura procesal (que podemos denominar como "dual" o "bifásica") es que el regulador analice siempre la concentración bajo un esquema deductivo, desde sus elementos más generales hacia los más particulares. De este modo, el organismo competente obtiene primero una "panorámica" de la fusión (primera etapa), luego de lo cual puede enfocarse única y exclusivamente en aquellos detalles que, a su juicio, mayores probabilidades tienen de generar efectos anticompetitivos (segunda etapa). Lo que se busca es que las operaciones pro-competitivas o neutras sean rápidamente aprobadas en primera etapa (ya sea en forma pura y simple o con condiciones negociadas con las partes) para que

\footnotetext{
9 Caviedes (2010).

10 Federal Trade Commission (2009), pp. 11-13; Office of Fair Trading (2009), pp. 1-19; Australian Competition And Consumer Commission (2008), pp. 20-31 y Consejo de Europa (2004).
} 
así el regulador pueda concentrarse únicamente en los aspectos cuestionables de las fusiones con serio riesgo anticompetitivo ${ }^{11}$.

Si se toma en cuenta que, como lo prueba la literatura económica existente a la fecha, la mayoría de las fusiones son procompetitivas o neutras, es necesario que sus procedimientos de control contemplen mecanismos de depuración o screening que permitan distinguir éstas de las operaciones anticompetitivas. En nuestro concepto, la estructura dual o bifásica genera eficientemente este descreme, ya que, por una parte, permite que los organismos competentes descarten rápidamente operaciones inofensivas o de bajo riesgo anticompetitivo (primera etapa); mientras que por otra ayuda a afinar el enfoque única y exclusivamente a aquellos elementos que, a juicio del regulador, tienen mayores probabilidades de generar efectos contrarios a la libre competencia (segunda etapa).

Por su parte, la Guía en comento establece un procedimiento especial, distinto de la consulta del art. 31 del D.L. № 211, que tiene una duración de hasta 60 días hábiles desde que se notifica a la Fiscalía, luego de lo cual ésta puede adoptar las siguientes resoluciones ${ }^{12}$ : a) archivar los antecedentes, lo que implica, en la práctica, aprobar la concentración, pero manteniendo la posibilidad de impugnarla ex post ${ }^{13}$; b) celebrar un acuerdo extrajudicial con las partes; o c) iniciar una consulta al Tribunal de Defensa de la Libre Competencia ("el Tribunal" o "TDLC").

Relacionando lo anterior, consideramos que es valorable que la Guía consagre este procedimiento previo puesto que (con las consideraciones del caso) éste podría hacer las veces de primera etapa que refine la cantidad de concentraciones que lleguen a consulta al TDLC. Empero, el que este procedimiento no sea obligatorio para las partes, y sobre todo el hecho que terceros tengan legitimación activa para iniciar la consulta, puede también conspirar fuertemente en contra del cumplimiento de ese objetivo.

\footnotetext{
${ }^{11}$ Las estadísticas ratifican la eficiencia de la estructura de dos etapas. En Estados Unidos, nada menos que un $67 \%$ de las concentraciones revisadas entre 1996 y 2006 fueron aprobadas con terminación anticipada, quedando el resto sujetas al procedimiento general; más aún, tan sólo el 2,7\% de estas concentraciones pasaron a la segunda etapa. Por otro lado, en el caso de la Unión Europea, tan sólo un 4,1\% de los 2.471 casos conocidos por la Comisión Europea en el período 2000-2007 pasaron a segunda etapa. Para revisar este y otros datos, ver GonZález y Benítez (2008).

12 Fiscalía Nacional Económica (2012), pp. 26-32.

13 Un importante caso anterior a la Guía en que se archivó la investigación, luego de que las partes cumpliesen con las exigencias planteadas por la FNE, fue el caso Cencosud/Johnson's (Corte Suprema, Rol No 1978-11 FNE (A)). Nehme y Mordoj (2012), p. 90.
} 


\subsection{Posibilidad de negociar ampliaciones a los plazos y medidas de mitigación ${ }^{14}$}

Además de lo anterior, otra clave de un buen sistema de control de fusiones es que tenga relativamente altos grados de flexibilidad y desformalización, por cuanto ello le permite al órgano competente adaptar fácilmente su análisis a las particularidades de cada operación. De hecho, si se revisa atentamente la normativa legal y reglamentaria norteamericana, británica, australiana y comunitaria europea, en todas ellas se le reconoce a la Administración relativamente altas dosis de discrecionalidad para disponer del procedimiento, al contrario de lo que sucede con la legislación procesal general. Para estos efectos, las leyes y reglamentos respectivos, en lugar de estar estructurados como "manuales de instrucciones" (que es la aproximación clásica) operan más bien como pautas limitativas y orientadoras de la discrecionalidad; pudiendo la autoridad incluso establecer "vías rápidas alternativas para aprobar operaciones con bajo riesgo anticompetitivo" ${ }^{15}$.

En definitiva, esta flexibilidad procesal funciona, al igual que la estructura dual, como un mecanismo depurador o descompresor del sistema, permitiéndole al regulador descartar rápidamente las concentraciones procompetitivas o neutras y focalizarse en aquellas con mayor riesgo anticompetitivo. Por estas razones, en los sistemas comparados el regulador recomienda siempre a las partes el llegar a acuerdo, recalcándoles además la conveniencia de que las condiciones se propongan al inicio del proceso y no en etapas intermedias o al final.

En relación a esto, es positivo que la Guía contemple la posibilidad de negociar tanto ampliaciones al plazo del procedimiento administrativo como las medidas de mitigación ${ }^{16}$, ya que ello beneficia tanto a las partes como a la Administración. En el caso del plazo, como los principales beneficiarios del mismo son las partes, es razonable que éstas puedan renunciar al mismo si lo estiman conveniente a sus intereses. Por otro lado, en el caso de las medidas de mitigación, el beneficio para la Administración es la eficiencia en la utilización de sus recursos (lo que a su vez permite focalizar el análisis a concentraciones

\footnotetext{
14 Para profundizar en el estudio de las medidas de mitigación, ver AgüERO (2010), pp. 313-323. Por otra parte, la función de las medidas de mitigación fue desarrollada recientemente por el TDLC en el caso John Malone (TDLC Rol C № 156-08, Sentencia № 117/2011), sosteniéndose, entre otras cosas, que: (i) ellas tienen duración indefinida a menos que se solicite su alzamiento en otro procedimiento no contencioso; (ii) su efecto es general o erga omnes; y (iii) son obligaciones de hacer o no hacer que pueden hacerse cumplir forzosamente ante tribunales. Nehme y Mordos (2012), p. 90.

1515 U.S.C. § 18a.; Office Of Fair Trading (2009), pp. 44-45; Australian Competition And Consumer Commission (2006), pp. 1-11 y Consejo de Europa (2004).

${ }^{16}$ Fiscalía Nacional EConómica (2012), pp. 26-32.
} 
riesgosas), mientras que el beneficio para la parte consiste en la rápida aprobación de la fusión, con grados de certeza jurídica a lo menos iguales a los que se generarían de no existir la posibilidad de negociar y con condiciones especialmente diseñadas a la misma que subsanen sus efectos anticompetitivos del modo menos gravoso posible.

\section{Lo que falta}

Habría sido deseable que la Guía contemplase también dos mecanismos de depuración o screening entre las operaciones sujetas a revisión, estos serían: a) umbrales referenciales y; b) un pliego de cargos o declaración de objeciones.

\section{a) Umbrales Referenciales}

Nuestro sistema de control de fusiones es voluntario, lo que significa que no existe obligación alguna de que las partes deban someter al conocimiento de los organismos reguladores de antimonopolios fusiones que superen ciertos umbrales prefijados por la ley.

Sin perjuicio de lo anterior, el hecho de que nuestro sistema sea voluntario no obsta a que, mientras no se efectúe una reforma legal que lo transforme en obligatorio, los organismos competentes establezcan por vía administrativa umbrales "referenciales" para consideración de las partes que pretendan efectuar una operación de concentración. A través de estos umbrales, la Administración básicamente envía una señal al mercado indicándole ex ante qué operaciones corresponde que sean notificadas por tener potencial efecto anticompetitivo.

A pesar de que pueda parecer extraña, una propuesta como esta no es tan exótica si se considera el sistema de control de fusiones británico. En efecto, aun cuando tal sistema es voluntario, la ley establece en forma clara y precisa una serie de umbrales (basados en el sistema de "ventas netas totales" o "utilidades netas totales") que tanto la Administración como los particulares deben tener en cuenta a la hora de determinar cuándo una operación tiene un potencial riesgo anticompetitivo que justifique notificar al regulador ${ }^{17}$. En consecuencia, incluso considerando que estos umbrales no son obligatorios, en el sistema británico ellos funcionan como criterios orientadores de la actividad administrativa destinados a otorgar certeza jurídica a los particulares.

Si relacionamos esto con la nueva Guía de la FNE, vemos que no se establecieron umbrales referenciales de ningún tipo. En efecto, la Guía únicamente afirma que, una vez hecha la notificación, el organismo caracterizará la operación, determinara el mercado relevante y aplicará el Índice de Herfindahl

17 Office of Fair Trading (2009), pp. 1-19. 
Hirschmann para determinar el grado de concentración del mismo, todo lo cual es positivo ${ }^{18}$. Pero más allá de eso no encontramos mención alguna a la etapa previa a la realización de todas estas operaciones, que es la determinación de si la fusión es susceptible de ser notificada o no.

En nuestro concepto, el establecimiento de umbrales basados en el criterio de ventas netas totales o utilidades netas totales, aun cuando hubiesen tenido un valor meramente referencial y sólo hubiesen sido incorporados por vía reglamentaria y no legal, habría permitido generar mayor certeza jurídica que la actualmente existente a las partes de una operación, junto con focalizar de mejor manera los recursos y personal de la Fiscalía a las operaciones que efectivamente fuesen notificadas bajo ese esquema.

\section{b) Pliego de cargos}

Como lo mencionamos anteriormente, en los sistemas comparados, cuando el regulador resuelve que una concentración debe pasar a la segunda etapa, emite un pliego de cargos o declaración de objeciones en que se indican solamente los aspectos cuestionables de la misma que serán analizados con mayor detalle ${ }^{19}$. Por contraste, la Guía no menciona en ninguna parte la emisión por parte de la Fiscalía de un documento de este tipo que detalle cuáles son las partes riesgosas de una concentración en contraposición a las inocuas ${ }^{20}$.

Incluso asumiendo que el procedimiento administrativo que establece la Guía no suple la posibilidad de consulta al TDLC (razón por la cual podría considerarse el inicio de una consulta como el símil chilensis del inicio de una segunda etapa), de todos modos habría constituido un avance que, en lugar de establecer un solo período de 60 días hábiles, la Fiscalía hubiese indicado dos períodos de 30 o 40 días hábiles cada uno. Por ejemplo, la Guía podría haber señalado que, transcurridos los primeros 30 o 40 días hábiles, la Fiscalía resolvería: a) archivar el caso; b) aprobar la fusión pura y simplemente o con medidas de mitigación negociadas; o c) emitir un pliego de cargos. Sólo en este último caso comenzaría una segunda fase de otros 30 o 40 días hábiles, luego de la cual la Fiscalía resolvería adoptando alguna de las alternativas que actualmente se encuentran en la Guía.

Las razones por las cuales sostenemos la conveniencia del pliego de cargos es la gran utilidad que presta este documento para distinguir los (en palabras de la propia Guía) "aspectos relevantes en torno al diagnóstico de los efectos que

\footnotetext{
18 Fiscalía Nacional Económica (2012), pp. 9-14.

19 Federal Trade Commission (2009), pp. 11-13; Office of Fair Trading (2009), pp. 1-19; Australian Competition And Consumer Commission (2008), pp. 20-31; y Consejo de Europa (2004).

20 Fiscalía Nacional Económica (2012), pp. 26-32.
} 
sobre la libre competencia habrá de tener la operación (... $)^{\prime 21}$ versus aquellos aspectos de menor importancia. En tal sentido, la incorporación expresa del pliego de cargos habría servido para orientar aún más claramente la actividad de los funcionarios de la FNE a la hora de considerar la negociación de medidas de mitigación o una eventual consulta al Tribunal, del mismo modo que también habría sido útil para las partes y a terceros, ya que les habría permitido evaluar tempranamente sus opciones frente al regulador.

\section{Lo que sólo el legislador puede solucionar}

Sin perjuicio de los aspectos mejorables de la Guía, existe una serie de problemas de nuestro sistema que solamente el legislador puede solucionar a través de una reforma al D.L. № 211.

\subsection{Voluntariedad vs obligatoriedad}

Existen dos tipos de sistemas de control de concentraciones: el sistema obligatorio y el sistema voluntario. En el primero, todas las operaciones de concentración que superen ciertos umbrales fijados por la ley deben notificarse al regulador antimonopolios, quien luego evaluará su carácter procompetitivo o anticompetitivo, aprobándola con o sin condiciones en el primer caso y rechazándola en el segundo. Por contraste, en el sistema voluntario no existe en principio obligación jurídica de notificar la fusión proyectada, pues la idea es que sólo se notifiquen fusiones que realmente tengan el potencial de producir efectos anticompetitivos para así focalizar los recursos públicos. Por supuesto, la eficacia del sistema voluntario opera sobre la base de que los incentivos estén bien colocados, de modo que efectivamente induzcan a las partes a notificar fusiones altamente riesgosas.

Una de las materias más investigadas por los especialistas en los últimos años es la de las ventajas y desventajas de cada uno de estos sistemas frente al otro. Hasta ahora, los resultados estarían demostrando que, por una parte, el sistema obligatorio tiene el mérito de otorgar mayores niveles de certeza jurídica, puesto que los agentes económicos conocerían ex ante exactamente cuándo notificar y cuándo no, siendo su inconveniente los riesgos de subnotificación o sobrenotificación de fusiones; mientras que por otra el sistema voluntario tendría el atributo de focalizar de mejor manera los recursos públicos, siendo su problema la colocación de incentivos para notificar cuando corresponde ${ }^{22}$.

${ }^{21}$ Fiscalía Nacional Económica (2012), p. 28.

22 González y Benítez (2008), pp. 1-11. 
La gran mayoría de los países y la Unión Europea cuentan con sistemas de notificación obligatoria. De hecho, de un total de cuarenta y ocho países, cuarenta y uno aplican el sistema obligatorio mientras que tan sólo siete (entre los que se encuentran Australia y el Reino Unido) aplican el sistema voluntario. Con todo, es necesario recalcar que los sistemas australiano y británico, aun cuando son la excepción, se encuentran catalogados dentro de los más eficientes a nivel mundial ${ }^{23}$.

Junto con los regímenes anteriormente descritos, en la actualidad se perfila el surgimiento de un tercer sistema, denominado sistema mixto, que consiste básicamente en un sistema obligatorio con la posibilidad de consultar operaciones cuyo resultado sea inferior a los umbrales de notificación. La idea es que este mecanismo se aplique en casos tales como, por ejemplo, el de una operación polémica y sometida a un fuerte escrutinio de los medios de comunicación social, pero que en principio no sería revisable por no quedar dentro de los umbrales de notificación del sistema obligatorio ${ }^{24}$.

En lo que respecta a Chile (que es de los pocos países con sistema voluntario $\left.^{25}\right)$, si ponderamos por una parte el relativamente escaso desarrollo del control de concentraciones, y por otra que uno de sus principales problemas es, en opinión de la doctrina, que los organismos competentes no han sido capaces de establecer criterios relativamente uniformes que entreguen seguridad jurídica ${ }^{26}$, concluimos que es necesario transformar el sistema chileno en obligatorio mediante una reforma al D.L. № 211. Ahora bien, reconocemos que el establecimiento de un sistema obligatorio genera el riesgo de sobrenotificación o subnotificación de concentraciones, sin embargo, estimamos que este riesgo puede ser fuertemente mitigado mediante el cálculo de los umbrales. Por esto, consideramos también que el criterio en virtud del cual deberían establecerse estos umbrales debería ser el de "ventas netas totales" o de "utilidades netas totales" en lugar del grado de concentración que tendría el agente económico

23 González y Benítez (2008), pp. 5-11.

24 Nehme y Mordos (2012), pp. 94-97.

25 Una de las situaciones que más críticas generó contra el carácter voluntario de nuestro sistema fue lo ocurrido con la fusión "Cencosud/Paris" cuando se la compara con lo que pasó en el caso "D \& S/ Falabella". Sucede que, si bien es cierto ambas concentraciones eran de una magnitud similar, la primera no se sometió a consulta y la segunda sí. El resultado de esto fue que, cuando se dictó la sentencia prohibiendo la fusión "D \& S/Falabella", el plazo para revisar la fusión Cencosud/Paris prescribió; todo lo cual significa que el sistema es capaz de premiar a quienes no consultan y castigar a quienes sí lo hacen. NeHme y Mordos (2012), p. 96.

26 Un caso en el cual quedan de manifiesto las desventajas del sistema voluntario es la fusión SMU/ Supermercados del Sur (Rol NC N $397-11$ ), en que se efectuó la consulta después de ejecutada la fusión y con el único objeto de que se evaluasen sus posibles medidas de mitigación. NeHME y MORDOJ (2012), p. 90. 
resultante de la operación, por cuanto es el más recomendado por la literatura económica especializada. Para efectos de incorporar la reajustabilidad, pensamos además que los umbrales deberían establecerse en unidades de fomento u otra unidad que establezca el Banco Central de Chile para reemplazarla.

\subsection{La subsistencia de la consulta para el tratamiento de fusiones}

Tal como señalamos supra, en nuestro derecho no existe un procedimiento pensado específicamente para el control de fusiones, ya que la consulta del art. 31 del D.L. № 211 sirve también para que el TDLC conozca otros hechos, actos o convenciones distintos a las fusiones ${ }^{27}$. Por otro lado, dijimos también que un aspecto fundamental del éxito de un procedimiento de control de concentraciones consiste en que se estructure en dos etapas, de manera que la primera funcione como un control preliminar de la operación en general y la segunda como un control focalizado a los aspectos problemáticos de la misma. Por estas razones (y por ser incompatible con la obligatoriedad sugerida en el punto anterior), estimamos necesario que el legislador establezca un procedimiento específico de revisión de concentraciones, distinto de la consulta, que reemplace tanto a ésta como a la nueva Guía y que rescate los aspectos positivos de este último documento.

Vinculado a esto, si se observan las características comunes de los sistemas norteamericano, británico, australiano y comunitario europeo, se puede derivar un modelo "estándar" que no varía demasiado por el hecho de que algunos de estos sistemas se estructuren bajo el modelo "agencia integrada" u otros bajo la categoría de "bifurcado judicial". En nuestro concepto, un procedimiento de estas características se estructuraría, a lo menos, por las siguientes etapas cronológicas: a) notificación; b) revisión de la fusión en primera etapa; c) resolución aprobando (con o sin condiciones) la operación, o transcurso de la primera etapa sin pronunciamiento; d) emisión de pliego de cargos a la fusión; e) revisión en segunda etapa de los aspectos objetados; f) nueva resolución de la Administración aprobando la operación (con o sin condiciones), rechazándola, o dejando que venza el plazo de la segunda etapa sin pronunciarse $y ;$ g) revisión de la resolución dictada por la autoridad competente en primera o en segunda fase, ya sea por otro órgano administrativo o por los tribunales.

En base a esta estructura, proponemos que la encargada de conocer la fusión en primera etapa sea la FNE. Preferimos este servicio al TDLC ya que, siendo la Fiscalía a quien corresponden las labores de monitoreo e investigación de los

27 De acuerdo a las estadísticas actualizadas del TDLC, el 25\% de las consultas realizadas son concentraciones, lo que las transforma en el segundo ítem más importante. El primero son las consultas por aplicación de resoluciones (32\%). Tribunal de Defensa de LA Libre Competencia (2013 b). 
delitos contra la libre competencia, consideramos que ella se encuentra mejor posicionada que el TDLC para hacer revisiones preliminares. Además, entregarle el conocimiento de la primera instancia al TDLC podría implicar para éste una sobrecarga de trabajo que distraiga su atención hacia operaciones poco riesgosas, lo que sería ineficiente. Todo lo anterior queda reafirmado si se toma en cuenta que, en derecho comparado, la primera etapa siempre es conocida por un órgano administrativo, lo que se explica por las ventajas de flexibilidad y especialización que éstos tienen frente a la judicatura.

El procedimiento se iniciaría con la notificación de las partes, quienes serían las únicas que tendrían legitimación activa para efectuarla. Recibida la notificación, la FNE la notificaría de inmediato al público. Luego, la Fiscalía tendría un plazo de aproximadamente 30 o 40 días hábiles para revisar, en primera fase, la fusión. Dentro de este plazo, la Fiscalía debería determinar si la operación: a) se aprueba pura y simplemente; b) se aprueba, sujeta a las condiciones que se negocien con las partes; o c) se objeta en determinados aspectos, los que deberían indicarse en forma taxativa en un pliego de cargos. Sólo en caso de que la concentración sea objetada se iniciaría la segunda etapa, en los demás operaría la inmunidad jurisdiccional respecto de las acciones judiciales de la Fiscalía (no de terceros), siempre que se cumplan las condiciones acordadas y los antecedentes aportados por las partes no sean falsos o engañosos. Las notificaciones al público se efectuarían a través del sistema establecido en el art. $31 \mathrm{~N}^{\circ} 1$ del D.L. No 211.

Durante la primera etapa, la FNE podría requerir de cualquier particular los antecedentes que considere necesarios para revisar la fusión (art. 39 letra h). Empero, en caso de que los requeridos impugnen esta solicitud de información al TDLC por estimar que irroga perjuicio a sus intereses o a los de terceros (inci$\operatorname{sos} 2^{\circ}$ a $4^{\circ}$ del precepto legal comentado), el transcurso del plazo de la primera etapa debería suspenderse hasta que el Tribunal dicte sentencia definitiva sobre el asunto. En cuanto a los terceros interesados, éstos podrían aportar al proceso todos los antecedentes que estimen convenientes, dentro de un plazo de aproximadamente quince o veinte días hábiles contados desde que se notifique el inicio del proceso.

Pero ¿qué pasaría si vence el plazo de la primera etapa y la Fiscalía no resuelve? En este caso, a menos que el plazo de revisión se amplíe producto de una negociación con las partes, debería operar el silencio administrativo positivo. Es decir, a falta de pronunciamiento de la Administración, la concentración debería entenderse aprobada por el solo ministerio de la ley con todas sus consecuencias, incluida, por cierto, la preclusión de acciones de la FNE. A petición de parte, la Fiscalía debería declarar la aplicación del silencio administrativo positivo sin más trámite, estando obligada además a notificar al público esta declaración. El motivo para preferir el silencio administrativo positivo al negativo es que, ya 
que la mayoría de las concentraciones son procompetitivas o neutras, parece razonable presumir la licitud de aquellas operaciones que no alcanzaron a ser revisadas por el regulador. El sistema comunitario europeo utiliza este criterio.

En la segunda etapa, que sólo se enfocaría en los aspectos objetados, hay dos alternativas para estructurar el procedimiento: la primera es que la fase sea conocida por la Fiscalía y la segunda es que este rol lo asuma el TDLC. Más allá de esto, en ambos casos el organismo tendría otros 30 o 40 días hábiles para analizar los elementos objetados, luego de lo cual debería resolver si: a) aprueba pura y simplemente la operación; (b) aprueba la operación con condiciones negociadas con las partes o; c) prohíbe la operación. En lo demás, se aplicarían las mismas normas de la primera etapa sobre participación de terceros, requerimientos de información a particulares, negociación de plazos y silencio administrativo positivo.

Recapitulando, la segunda etapa podría ser conocida tanto por la FNE como por el TDLC. Sin embargo, hay dos grandes diferencias que se producirían de entregarle la competencia respectiva a un organismo en lugar del otro. La primera dice relación con el grado de flexibilidad procedimental de la revisión, mientras que la segunda se refiere a la amplitud de las posibilidades de impugnar judicialmente la actuación administrativa. En cuanto a lo primero, por sus propias características institucionales, una segunda etapa conocida por la Fiscalía tiene mayores posibilidades de regularse por un procedimiento flexible y desformalizado que una conocida por el TDLC, ya que, en nuestro derecho, este último órgano es un tribunal, lo que necesariamente se traduce en que cumpla con mayores estándares de independencia e imparcialidad que implican mayor formalismo en el análisis del caso.

En cuanto a lo segundo, si la segunda etapa fuese conocida por la FNE, la revisión judicial de la actuación de este servicio público correspondería, en principio, al TDLC en primera instancia vía revisión, y a la Corte Suprema en segunda instancia vía apelación. Por el contrario, si fuese al tribunal al que le correspondiese conocer la segunda etapa, la revisión judicial de sus actos recaería únicamente en la Corte Suprema, lo que haría que el procedimiento fuese más semejante, en este aspecto, a la consulta que actualmente existe. Ergo, de entregarse el conocimiento de la segunda etapa al TDLC, las posibilidades de revisión judicial serían menores que si se le confiriese esta potestad a la FNE. Por tanto, para escoger si la segunda etapa debe ser conocida por la FNE o por el TDLC hay que ponderar, por un lado, la ventaja que implica la flexibilidad procesal de la Fiscalía versus la menor revisión judicial (con la consiguiente mayor rapidez) que supone optar por el Tribunal como revisor no contencioso.

A nuestro juicio, la segunda etapa debería ser conocida por la FNE aún a riesgo de incrementar las posibilidades de revisión judicial. Elegimos esta 
alternativa puesto que la ductilidad procesal es clave para el desempeño del sistema, ya que permite adaptar el análisis a la casuística de cada fusión. Por otro lado, el incremento de las oportunidades de revisión judicial depende no solamente del número de tribunales, sino también del mayor o menor alcance que tengan los recursos para impugnar sentencias, es decir, las posibilidades de revisión judicial cambian sustancialmente si se reconoce la posibilidad de apelar, de manera que el TDLC sea el tribunal de primera instancia y la Corte Suprema el de alzada, en contraposición a que se establezca al TDLC como tribunal de única instancia, con la posibilidad de recurrir extraordinariamente a la Corte Suprema.

Con el objeto de evitar que la proliferación de medios de impugnación se traduzca en dilaciones excesivas, optamos por que se establezca al TDLC como tribunal de única instancia, pero con la posibilidad de recurrir la sentencia ante la Corte Suprema mediante un recurso extraordinario de "nulidad" o "casación ampliada", limitado exclusivamente a la revisión del derecho. Preferimos esta alternativa por cuanto existen buenos argumentos jurídicos y de política pública a favor de la limitación o eliminación del recurso de apelación ${ }^{28}$.

A mayor abundamiento, el establecimiento de un sistema de revisión judicial como el propuesto generaría un contencioso administrativo de libre competencia más coherente con el resto de las reformas procesales aplicadas en Chile y actualmente en curso. En efecto, en las principales reformas ejecutadas a la fecha, existe la intención de fortalecer el rol de la Corte Suprema como un tribunal de casación uniformador de la jurisprudencia, descargándola en consecuencia de otro tipo de tareas. Esta opción se alinea claramente con esta tendencia, que es la misma que adopta el sistema europeo.

\subsection{Flexibilidad procesal}

Como indicamos precedentemente, un aspecto fundamental de un buen procedimiento de control de fusiones es que sea relativamente flexible y desformalizado, lo que se manifiesta en: a) posibilidad de que la autoridad establezca diferentes tipos de formularios de notificación dependiendo de la complejidad de la operación; b) posibilidad de negociar, durante todo el proceso, medidas de mitigación con las partes; c) posibilidad de establecer, por vía administrativa, procedimientos alternativos (Ej. Australia y Reino Unido); d) posibilidad de disponer de los plazos, de oficio o con acuerdo de las partes; e) posibilidad de determinar si se efectúan o no reuniones con las partes, junto con el número y materias a tratar en las mismas; f) posibilidad de declarar la confidencialidad o

${ }^{28}$ Vargas y Fuentes (2011), pp. 341-366; Luco e Ibarra (2011), pp. 419-434 y Leturia y Caviedes (2011), pp. 401-418. 
reserva de documentos; g) posibilidad de requerir de las partes o de terceros la presentación de antecedentes, so pena de aplicarles una sanción penal o administrativa, junto con la atribución para ordenar la realización de diligencias probatorias, tales como declaraciones de partes y de testigos y; h) posibilidad de determinar la celebración de audiencias, pudiendo decidir acerca de su nivel de publicidad y sobre la participación de terceros ${ }^{29}$.

En el caso chileno, abogamos por que se le otorguen a la Fiscalía similares potestades para disponer de ciertos elementos procesales. En concreto, y en conjunto con las atribuciones que actualmente detenta, a la Fiscalía deberían otorgársele las siguientes potestades: a) establecer varios tipos de formularios de notificación dependiendo de la fusión a revisar. La idea es que los formularios más detallados se apliquen a las operaciones con mayor riesgo anticompetitivo, mientras que los otros ("simplificados" o "abreviados") sean utilizados para concentraciones menos riesgosas; b) negociar con las partes ampliaciones a los plazos de revisión; y c) facultad de convocar a las partes y a los terceros a una o más audiencias, de manera que ellos puedan exponer oralmente sus posiciones respectivas. Sin embargo, aquí hay que distinguir entre la situación de las partes y la de los terceros.

En el caso de las partes, ellas deberían tener el derecho de solicitar a la Fiscalía la realización de una audiencia oral durante la segunda etapa, caso en el cual ésta se encontraría obligada a convocarla. Es decir, las partes tendrían siempre el derecho a "su día ante el Fiscal", sin perjuicio de que además se celebren otras audiencias por iniciativa del organismo. Por el contrario, estimo que la participación de terceros en audiencias orales no debe ser un derecho subjetivo, sino que tiene que estar supeditada a la discrecionalidad de la FNE. A continuación desarrollamos este último argumento.

\subsection{Limitar las posibilidades de participación de terceros}

Nuestro legislador, con el propósito de ampliar la revisión no contenciosa del TDLC, estableció a través de la Ley № 20.361 la posibilidad de que cualquier legítimo interesado (dentro de los cuales se ha incluido, por vía jurisprudencial, a entidades tales como las Asociaciones de Consumidores), pueda iniciar el procedimiento de consulta del artículo 31 del D.L. № $211^{30}$. A contrario sensu, en ninguno de los principales sistemas comparados de control de fusiones se permite que terceros interesados puedan iniciar la revisión.

2915 U.S.C. $\S 18$ a.; Office of Fair Trading (2009), pp. 44-45; Australian Competition and Consumer Commission (2006), pp. 1-11 y Consejo de Europa (2004).

30 Nehme y Mordos (2012), pp. 104-113. 
Más aún, la regla general es que la intervención de dichos terceros se limite únicamente a: a) el aporte de antecedentes; b) la intervención en las audiencias respectivas, sujetos a la aquiescencia del regulador y; c) la impugnación de la resolución del órgano administrativo que revisó la operación, sólo en cuanto se acredite agravio.

De acuerdo a Nehme y Mordoj ${ }^{31}$, que los terceros puedan iniciar una consulta es contraproducente con los objetivos del sistema, ya que permite que ellos adopten comportamientos oportunistas notificando incluso operaciones que no presentan riesgo anticompetitivo. En opinión de estos autores, todo esto incrementa la inseguridad jurídica, ya que obliga a las partes y a la FNE a internalizar los costos de una potencial consulta de terceros sobre todo en aquellos casos en que tienen la intención de llegar a acuerdo. Compartimos plenamente estas apreciaciones ${ }^{32}$.

Por estas razones, cualquier modificación que se haga al sistema chileno debería excluir completamente la legitimación activa de terceros. En cuanto a las demás formas de intervención, hay que distinguir entre: a) la posibilidad de presentar antecedentes, que se debiese mantener en la primera y en la segunda etapa y; b) la participación en audiencias orales, que no debería ser un derecho de los terceros sino que depender de la discreción de la FNE. Sostenemos esto último porque, existiendo la posibilidad de que los terceros presenten previamente sus documentos, no es indispensable su intervención en una audiencia, más aún si se aprecia que, por la complejidad técnica que implica la revisión de una fusión, la gran mayoría de tales antecedentes constan por escrito. En estas circunstancias, reconocerle indiscriminadamente a los terceros la posibilidad de intervenir en audiencias orales daría pábulo para que simplemente se obstaculice la operación, sin que ello necesariamente signifique un aporte de información relevante para la Fiscalía.

Por último, en cuanto a la legitimación activa de los terceros interesados para impugnar judicialmente la resolución administrativa que recaiga sobre la fusión, nuestra posición es que ella debe mantenerse, pero encuadrándose únicamente en las hipótesis de que la concentración se apruebe (con o sin medidas de mitigación), o que opere el silencio administrativo positivo, y siempre que se acredite agravio.

\footnotetext{
31 Nehme y Mordoj (2012), pp. 104-113.

32 A nuestro juicio, el caso Lan/Tam (Rol NC No 388-11) es el paradigma de los problemas que implica otorgarle a terceros la facultad de iniciar los procesos de consulta. Como es sabido, en este caso la FNE y las compañías involucradas Ilegaron a un acuerdo extrajudicial; sin embargo, este acuerdo fue frustrado por una consulta efectuada por una organización de consumidores, Ilamada ConADECUS. Nehme y Mordoj (2012), pp. 89-92.
} 


\section{BiBLIOGRAFíA CitADA}

\section{FUENTES DIGITALES}

Australian Competition and Consumer Commission (2008): Formal Merger Review Process Guidelines. Disponible en: http://www.accc.gov.au/content/index. phtml/itemld/776536 [visitado el 21/04/2012].

Australian Competition and Consumer Commission (2006): Merger Review Process Guidelines. Disponible en: http://www.accc.gov.au/content/index.phtml/ itemld/776536 [visitado el 21/04/2012].

Caviedes, Cristóbal (2010): Sistemas de Control Preventivo de Fusiones en Derecho Comparado. Disponible en: http://tesis.uchile.cl/handle/2250/113019 [visitado el 02/10/2013].

Consejo de Europa (2004): Reglamento del consejo No 139/2004. Disponible en: http://eur-lex.europa.eu/LexUriServ/LexUriServ. do?uri=CELEX:32004R0139:ES:HTML [visitado el 20/05/2012].

Fiscalía Nacional Económica (2012): Guía para el Análisis de Operaciones de Concentración. Disponible en: http://www.fne.gob.cl/wp-content/ uploads/2012/10/Guia-Fusiones.pdf [visitado el 25/10/2012].

Federal Trade Commission (2009): What is the Premerger Notification Program? Disponible en: http://www.ftc.gov/bc/hsr/introguides/introguides.shtm [visitado el 15/11/2011].

González, Aldo y Benítez, Daniel (2008): Pre-Merger Notification Mechanisms: Incentives and Efficiency of Mandatory and Voluntary Mechanisms. Disponible en: http://www.sociedadpoliticaspublicas.cl/archivos [visitado el 29/02/2012].

Office of Fair Trading (2009): Mergers Jurisdictional and Procedural Guidance. Disponible en: http://www.oft.gov.uk/OFTwork/mergers/publications/ [visitado el 17/11/2011].

Tribunal de Defensa de la Libre Competencia (2013 a): Causas No ContenciosasDuración Promedio. En: http://www.tdlc.cl/Portal.Base/Web/VerContenido. aspx?GUID=\&ID=341 [visitado el 13/01/2013].

Tribunal de Defensa de la Libre Competencia (2013 b): Causas No Contenciosas Objeto de las Consultas Presentadas al TDLC. Disponible en: http://www. tdlc.cl/Portal.Base/Web/VerContenido.aspx?GUID=\&ID=341 [visitado el 13/01/2013].

\section{ARtí́CULOS DE PUbliCACIONES PERIÓDICAS}

Nehme, Nicole y MordoJ, Benjamín (2012): "Diseños Institucionales de Control de Concentraciones: Análisis y Propuestas", en: Estudios Públicos ( $\left.\mathrm{N}^{\circ} 125\right)$, pp. 87-137. 


\section{CAPÍTULOS DE LIBROS}

AgüERO, Francisco (2010): “Rol de las Medidas de Mitigación o Condiciones Impuestas para Aprobar Operaciones de Concentración: Un Análisis Aplicado al Sector Retail", en: Libre Competencia y Retail: Un Análisis Crítico (Santiago, Ed. LegalPublishing), pp. 313-323.

Leturia, Francisco Javier y CaVIedes, Cristóbal (2011): “La Restricción de la Doble Instancia en el Procedimiento Civil: Sistematización y Síntesis de sus Argumentos", en: Justicia Civil y Comercial: Una Reforma ¿Cercana? (Santiago, Ed. Libertad y Desarrollo), pp. 401-418.

Luco, Nicolás e IBARRA, Manuel (2011): “¿Es Compatible una Doble Instancia con los Principios de Oralidad, Inmediación y Concentración?", en: Justicia Civil y Comercial: Una Reforma ¿Cercana? (Santiago, Ed. Libertad y Desarrollo), pp. 419-434.

VARGAS, Juan Enrique y Fuentes, Claudio, "Régimen Recursivo para la Reforma a la Justicia Civil", en: Justicia Civil y Comercial: Una Reforma ¿Cercana? (Santiago, Ed. Libertad y Desarrollo), pp. 341-366.

NORMAS JURÍDICAS CITADAS

Decreto con Fuerza de Ley No 1 del Ministerio de Economía, Fomento y Reconstrucción, Fija el Texto Refundido, Coordinado y Sistematizado del Decreto Ley № 211 de 1973, Diario Oficial, 7 de marzo de 2005.

15 U.S.C. § 18a. Disponible en: http://www.law.cornell.edu/uscode/text[visitado el 15/11/2011]. 